\title{
Microbiological methods to detect intestinal carriage of highly-resistant microorganisms (HRMO) in humans and livestock in the i-4-1-Health Dutch- Belgian cross-border project
}

\author{
Marjolein Kluytmans-van den Bergh ${ }^{1,2,3}$, Christine Lammens ${ }^{4}$, Natascha Perales Selva ${ }^{5}$, Carlo \\ Verhulst $^{1,6}$, Anton Buiting ${ }^{7}$, Isabel Leroux-Roels ${ }^{8}$, Veroniek Saegeman ${ }^{9}$, Paul Savelkoul ${ }^{10}$, Annette \\ Schuermans ${ }^{9}$, Jacobien Veenemans ${ }^{11}$, Dewi van der Vegt ${ }^{12}$, Ina Willemsen ${ }^{1}$, Bas Wintermans ${ }^{11}$, \\ Herman Goossens ${ }^{4,5}$, Jan Kluytmans ${ }^{1,3,6}$, and the i-4-1-Health Study Group
}

1 Department of Infection Control, Amphia Hospital, Breda, the Netherlands

2 Amphia Academy Infectious Disease Foundation, Amphia Hospital, Breda, the Netherlands

3 Julius Center for Health Sciences and Primary Care, University Medical Center Utrecht, Utrecht University, Utrecht, the Netherlands

4 Laboratory of Medical Microbiology, Vaccine \& Infectious Disease Institute, University of Antwerp, Antwerp, Belgium

5 Laboratory of Clinical Microbiology, Antwerp University Hospital, Antwerp, Belgium

6 Microvida Laboratory for Medical Microbiology, Amphia Hospital, Breda, the Netherlands

7 Laboratory for Medical Microbiology and Immunology, Elisabeth-TweeSteden Hospital, Tilburg, the Netherlands

8 Department of Laboratory Medicine, Ghent University Hospital, Ghent, Belgium

9 Department of Infection Control, University Hospitals Leuven, Leuven, Belgium

10 Department of Medical Microbiology, School for Public Health and Primary Care (CAPHRI), Maastricht University Medical Center+, Maastricht, the Netherlands

11 Laboratory for Microbiology, Admiraal De Ruyter Hospital, Goes, the Netherlands

12 PAMM Laboratory for Pathology and Medical Microbiology, Veldhoven, the Netherlands

\section{Keywords}

Antimicrobial resistance, intestinal carriage, detection, extended-spectrum beta-lactamase, carbapenem resistance, colistin resistance, ciprofloxacin resistance, vancomcin resistance, Enterobacterales, enterococci

\section{Corresponding author}

Marjolein F.Q. Kluytmans-van den Bergh

Department of Infection Control

Amphia Hospital, Breda, the Netherlands

E: marjoleinkluytmans@gmail.com 


\begin{abstract}
The i-4-1-Health project is a One Health project on the prevalence and spread of antimicrobial resistance in the human and veterinary domain in the Dutch-Belgian cross-border region. This paper describes the main components of the laboratory protocol that was developed to standardise the microbiological methods used for the detection of intestinal carriage of highly-resistant microorganisms.
\end{abstract}

\title{
INTRODUCTION
}

The global emergence and spread of antimicrobial resistance pose a critical threat to public health and livestock agriculture. The understanding and control of antimicrobial resistance is a complex global health challenge and asks for One Health approaches at both national and international levels. ${ }^{1-4}$ The i-4-1-Health project aims to address gaps in current knowledge and understanding of the complex routes of dissemination of antibiotic resistance by taking a One Health approach that includes both the human and veterinary domain and targets antibiotic use, infection control and antimicrobial resistance in the Dutch-Belgian cross-border region. Data are collected in hospitals, nursing homes, daycare centres, primary schools, and livestock farms. To the best of our knowledge, this is the first project to study intestinal carriage of a wide range of antimicrobial-resistant microorganisms in the human and veterinary domain in a cross-border setting.

This laboratory protocol was developed by the participating laboratories to standardise the microbiological methods used for the detection of intestinal carriage of highly-resistant microorganisms (HRMO), including extended-spectrum beta-lactamase-producing Enterobacterales (ESBL-E), carbapenem-resistant Gram-negative bacteria (carbaR-GNS), colistin-resistant Enterobacterales (coliR-E), ciprofloxacin-resistant Enterobacterales (ciproR-E) and vancomycin-resistant enterococci (VRE).

\section{METHODS}

\section{Study period and setting}

The i-4-1-Health study is performed in the Dutch-Belgian border region, comprising eight provinces (Figure 1). From October 2017 through January 2019, an anticipated number of 16,200 microbiological samples is collected in nine hospitals, 20 nursing homes, 20 daycare centres, 20 primary schools, 30 broiler farms and 30 multiplier pig farms in the Dutch-Belgian border region (Figure 2). Data are collected in cross-sectional surveys; three rounds of repeated surveys in hospitals, a single survey in nursing homes, daycare centres and primary schools, and three repeated surveys in livestock farms.

\section{Laboratories}

Microbiological cultures are processed in nine microbiology laboratories: 1) Laboratory of Medical Microbiology, Vaccine \& Infectious Disease Institute, University of Antwerp, Antwerp, Belgium; 2) Laboratory of Clinical Microbiology, Antwerp University Hospital, Antwerp, Belgium; 3) Department of Laboratory Medicine, Ghent University Hospital, Ghent, Belgium; 4) Department of Laboratory 
Medicine, University Hospitals Leuven, Leuven, Belgium; 5) Microvida Laboratory for Medical Microbiology, Amphia Hospital, Breda, the Netherlands; 6) Laboratory for Microbiology, Admiraal De Ruyter Hospital, Goes, the Netherlands; 7) Department of Medical Microbiology, Maastricht University Medical Center+, Maastricht, the Netherlands; 8) Laboratory for Medical Microbiology and Immunology, Elisabeth-TweeSteden Hospital, Tilburg, the Netherlands; and 9) PAMM Laboratory for Pathology and Medical Microbiology, Veldhoven, the Netherlands.

\section{Microbiological methods}

Microbiological methods are standardised across the nine participating laboratories (Figure 3). Nylonflocked swabs with $2 \mathrm{ml}$ Cary-Blair medium (FecalSwab ${ }^{\circledR}$, Copan Italy, Brescia, Italy) are used for collection and transport of faecal, perianal or gastrointestinal stoma samples. Swabs are kept at 2 to $8^{\circ} \mathrm{C}$, transported at room temperature and processed within 72 hours after sampling. Swabs that are not processed within 72 hours are rejected. A Columbia (5\%) sheep blood agar plate (bioMérieux, Marcy l'Etoile, France) is inoculated with $10 \mathrm{ul}$ of the Cary-Blair medium and serves as growth control. Swabs are rejected when the blood agar plate shows no bacterial growth, which is judged to be indicative of inappropriate sampling. The remaining Cary-Blair medium is used to inoculate $5 \mathrm{~mL}$ of a non-selective tryptic soy broth (TSB) (Copan Italy, Brescia, Italy). The blood agar plate and the TSB are incubated for 18 to 24 hours at 35 to $37^{\circ} \mathrm{C}$. Subsequently, $10 \mathrm{ul}$ of the TSB is subcultured on four commercially available selective agar plates, i.e. ChromID ${ }^{\circledR}$ ESBL, ChromID ${ }^{\circledR} \mathrm{CARBA} \mathrm{ChromID}^{\circledR}$ OXA-48 and (for human samples) ChromID ${ }^{\circledR}$ VRE (bioMérieux, Marcy l'Etoile, France). In three laboratories (University of Antwerp, Antwerp University Hospital, Amphia Hospital), two additional inhouse selective agar plates are used, i.e. McC ciprofloxacin, a MacConkey agar plate (Oxoid, Basingstoke, United Kingdom) with ciprofloxacin (2 mg/L; Sigma-Aldrich, Saint Louis, United States) and EMB colistin, an eosin methylene blue agar plate (Oxoid, Basingstoke, United Kingdom) with colistin (3.5 mg/L; Sigma-Aldrich, Saint Louis, United States), daptomycin (10 mg/L) and amphoterin B $(5 \mathrm{mg} / \mathrm{L}) .{ }^{5}$ Selective agar plates are incubated at 35 to $37^{\circ} \mathrm{C}$ for 18 to 24 hours, except for the ChromID ${ }^{\circledR}$ VRE which is incubated for 42 to 48 hours. The remaining TSB is stored at $-80^{\circ} \mathrm{C}$ for future (molecular) analyses.

The methods used for microbial identification and antimicrobial susceptibility testing are dependent on local routine laboratory practice (Table 1). Automated mass spectrometry systems with MALDI-TOF technology are used for microbial identification, i.e. either the VITEK ${ }^{\circledR}$ MS system (bioMérieux, Marcy l'Etoile, France) or the MALDI Biotyper ${ }^{\circledR}$ (Bruker Daltonic, Bremen, Germany). Antimicrobial susceptibility testing is performed with the semi-automated systems VITEK ${ }^{\circledR} 2$ (AST card N344 and P586) (bioMérieux, Marcy l'Etoile, France) or BD Phoenix ${ }^{\text {TM }}$ (AST card NMIC-417 and PMIC-96) (Becton Dickinson, Sparks, MD, United States) or with the disc diffusion method (Neo-Sensitabs ${ }^{\mathrm{TM}}$, Rosco, Taastrup, Denmark; Bio-Rad ${ }^{\circledR}$, Bio-Rad Laboratories, Marnes-la-Coquette, France; or BD BBL $^{T M}$ Sensi-Disc ${ }^{\mathrm{TM}}$, Becton Dickinson, Sparks, MD, United States) on Mueller Hinton E agar plates (MHA) (bioMérieux, Marcy l'Etoile, France) (Table 2 and Table 3). Reading of disc diffusion susceptibility tests is performed either manually or with an automated zone reader (SIRscan, i2a 
Diagnostics, Montpellier, France; ProtoCOL 3, Synbiosis, Cambridge, United Kingdom; or AGADIO'M , Bio-Rad Laboratories, Marnes-la-Coquette, France).

In addition, for Enterobacterales that grow on the EMB colistin agar plate, the MIC for colistin is determined with the MICRONAUT MIC-strip Colistin, a broth microdilution method with Mueller Hinton cation adjusted broth (Merlin Diagnostika, Bornheim, Germany). ${ }^{6}$ For enterococci that grow on the ChromID ${ }^{\circledR}$ VRE, the MICs for vancomycin and teicoplanin are determined with the gradient test method ETEST ${ }^{\circledR}$ (bioMérieux, Marcy l'Etoile, France). MICs and disk diffusion inhibition zones are interpreted using EUCAST breakpoint tables, version 8.1.7 For Enterobacterales that grow on the ChromID ${ }^{\circledR} E S B L$, the production of ESBL is phenotypically confirmed by the combination disk diffusion method with cefotaxime (30 ug), ceftazidime (30 ug), and cefepime ( $30 \mathrm{ug})$, each alone and combined with clavulanic acid (10 ug) (Neo-Sensitabs ${ }^{\mathrm{TM}}$, Rosco, Taastrup, Denmark) on MHA plates (bioMérieux, Marcy l'Etoile, France). Test results are interpreted according to EUCAST guidelines for detection of resistance mechanisms (Table 4). ${ }^{8}$ All antimicrobial-resistant bacterial isolates are stored at $-80^{\circ} \mathrm{C}$ using cryobeads or a cryopreservative glycerol broth. An anticipated number of 2,600 highlyresistant bacterial isolates is selected for whole-genome sequencing to identify genetic markers for antimicrobial resistance, clonal relatedness of isolates within and between domains and horizontal transfer of resistance genes.

\section{Quality control}

The following isolates are used for quality control: E. coli ATCC 25922, E. faecalis ATCC 29212, K. pneumoniae ATCC 700603 (SHV-18), K. pneumoniae ATCC BAA1705 (KPC, ciprofloxacin resistant), K. pneumoniae NCTC 13442 (OXA-48), E. coli NCTC 13846 (MCR-1), E. faecium CCUG 36804 (vanA), and E. faecalis ATCC 51299 (vanB).

\section{Data labelling}

The labelling system is designed to ensure that data are collected, stored and accessible in a way that permits cross-border and cross-domain analyses. Unique study numbers are generated for each human and veterinary sample included in the study. Each study number contains unique numeric codes for 'country', 'domain', 'institution or farm', 'ward, group or stable', 'survey' and a serial number (Figure 4). The study number used for the collection of microbiological data is identical to that used for the collection of demographic and clinical data. Labels with study numbers are pre-printed to avoid errors of transcription and interpretation with hand-written labels. For each ward or stable, one sheet with all labels for the ward or stable is prepared.

Unique isolate numbers are generated for each antimicrobial-resistant bacterial isolate stored. Each isolate number contains a unique numeric code for 'laboratory' and a serial number (Figure 4).

\section{Data storage}

Microbiological culture results are recorded in the local laboratory information system. At regular intervals, data are transferred to the central database where the data undergo final cleaning and are prepared for analyses. 


\section{Ethics}

For the human domain, the study protocol was reviewed by the Medical Research and Ethics Committee of the University Medical Center Utrecht, Utrecht, the Netherlands (Protocol Number 17426/C), the Medical Research and Ethics Committee of the Maastricht University Medical Center+ (Maastricht, the Netherlands) (METC 2017-0115 and METC 2017-0116), the Ethics Committee of the University Hospital Antwerp, Antwerp, Belgium (Belgian Registration Number B300210733784), the Ethics Committee of the Ghent University Hospital, Ghent, Belgium (Belgian Registration Number B670201733428), and the Ethics Committee of the University Hospitals Leuven, Leuven, Belgium (S59580 BD1 and S61807). The study was judged to be beyond the scope of the Dutch Medical Research Involving Human Subjects Act and the Belgian Law on Experiments on Humans, dated May 7th, 2004. Written or verbal informed consent for data collection and taking a faecal, perianal or gastrointestinal stoma swab for microbiological culture is obtained from all participants or their legal representatives.

For the veterinary domain, approval by an animal welfare body is not required. The procedure to collect fresh faecal droppings is considered to cause no discomfort, and animals are neither handled nor sacrificed during the study (EC Directive 2010/63).

All human data are anonymised, i.e. data cannot be directly or indirectly related to their source. Data on institutions and farms are pseudonymised, i.e. identifying information is replaced by a code, and a key file that links this code to the identifying information is kept separate from the research data.

\section{Data sharing}

During the study, feedback of resistance data is provided in-between surveys in hospitals and livestock farms. All investigators will have access to the final datasets and bacterial isolate collections to the extent necessary for answering research questions that are included as such in the i-4-1-Health publication plan. The findings will be presented at national and international conferences and submitted to peer-reviewed journals for publication. Publications are anticipated from 2019 and onwards. No earlier than December 31 st, 2020 and no later than December 31st, 2024, the final datasets and isolate collections will be made available in accordance with the FAIR (Findable, Accessible, Interoperable and Reusable) data principles. ${ }^{9}$

\section{DISCUSSION}

\section{Strengths}

- The i-4-1-Health project takes a One Health approach to address gaps in current knowledge and understanding of the complex routes of dissemination of antibiotic resistance.

- It is the first project to study intestinal carriage of a wide range of antimicrobial-resistant microorganisms in the human and veterinary domain in a cross-border setting using uniform methodology across domains.

- Repeated measurements in hospitals and livestock farms make it possible to study trends over time. 
- The use of pre-enrichment and selective agar plates increases the sensitivity of the methods to detect intestinal carriage of HRMO. ${ }^{10,11}$

\section{Limitations}

- The human domain includes patients in hospitals, nursing home residents and children in primary school and daycare centres, but not adults in the general population.

- The veterinary domain includes broiler farms and multiplier pig farms, but not non-chicken poultry farms, finishing pig farms and veal farms.

\section{ACKNOWLEDGEMENTS}

We are grateful to the collaborators in the participating laboratories, hospitals, nursing homes, primary schools, daycare centres and livestock farms for their contribution to the collection of the microbiological and epidemiological data.

\section{Funding}

The i-4-1-Health project is financed by the Interreg V Flanders-The Netherlands program, the crossborder cooperation program with financial support from the European Regional Development Fund (ERDF). Additional financial support is received from the Dutch Ministry of Health, Welfare and Sport, the Dutch Ministry of Economic Affairs, the Province of Noord-Brabant, the Belgian Department of Agriculture and Fisheries, the Province of Antwerp and the Province of East-Flanders. Selective and non-selective agar plates, ETEST ${ }^{\circledR}$ strips and VITEK ${ }^{\circledR} 2$ AST cards are provided by bioMérieux (Marcy I'Etoile, France); FecalSwabs ${ }^{\circledR}$ and tryptic soy broths are provided by Copan Italy (Brescia, Italy). The authors are free to publish the results from the project without interference from the funding bodies, bioMérieux or Copan Italy.

\section{i-4-1-Health Study Group}

Lieke van Alphen (Maastricht University Medical Center+, Maastricht, the Netherlands), Nicole van den Braak (Avans University of Applied Sciences, Breda, the Netherlands), Caroline Broucke (Agency for Care and Health, Brussels, Belgium), Anton Buiting (Elisabeth-TweeSteden Hospital, Tilburg, the Netherlands), Liselotte Coorevits (Ghent University Hospital, Ghent, Belgium), Sara Dequeker (Agency for Care and Health, Brussels, Belgium and Sciensano, Brussels, Belgium), Jeroen Dewulf (Ghent University, Ghent, Belgium), Wouter Dhaeze (Agency for Care and Health, Brussels, Belgium), Bram Diederen (ZorgSaam Hospital, Terneuzen, the Netherlands), Helen Ewalts (Regional Public Health Service Hart voor Brabant, Tilburg, the Netherlands), Herman Goossens (University of Antwerp, Antwerpen, Belgium and Antwerp University Hospital, Antwerp, Belgium), Inge Gyssens (Hasselt University, Hasselt, Belgium), Casper den Heijer (Regional Public Health Service ZuidLimburg, Heerlen, the Netherlands), Christian Hoebe (Maastricht University Medical Center+, Maastricht, the Netherlands and Regional Public Health Service Zuid-Limburg, Heerlen, the 
Netherlands), Casper Jamin (Maastricht University Medical Center+, Maastricht, the Netherlands), Patricia Jansingh (Regional Public Health Service Limburg Noord, Venlo, the Netherlands), Jan Kluytmans (Amphia Hospital, Breda, the Netherlands and University Medical Center Utrecht, Utrecht University, Utrecht, the Netherlands), Marjolein Kluytmans-van den Bergh (Amphia Hospital, Breda, the Netherlands and University Medical Center Utrecht, Utrecht University, Utrecht, the Netherlands), Stefanie van Koeveringe (Antwerp University Hospital, Antwerp, Belgium), Sien De Koster (University of Antwerp, Antwerp, Belgium), Christine Lammens (University of Antwerp, Antwerp, Belgium), Isabel Leroux-Roels (Ghent University Hospital, Ghent, Belgium), Hanna Masson (Agency for Care and Health, Brussel, Belgium), Ellen Nieuwkoop (Elisabeth-TweeSteden Hospital, Tilburg, the Netherlands), Anita van Oosten (Admiraal De Ruyter Hospital, Goes, the Netherlands), Natascha Perales Selva (Antwerp University Hospital, Antwerp, Belgium), Merel Postma (Ghent University, Ghent, Belgium), Stijn Raven (Regional Public Health Service West-Brabant, Breda, the Netherlands), Veroniek Saegeman (University Hospitals Leuven, Leuven, Belgium), Paul Savelkoul (Maastricht University Medical Center+, Maastricht, the Netherlands), Annette Schuermans (University Hospitals Leuven, Leuven, Belgium), Nathalie Sleeckx (Experimental Poultry Centre, Geel, Belgium), Arjan Stegeman (Utrecht University, Utrecht, the Netherlands), Tijs Tobias (Utrecht University, Utrecht, the Netherlands), Paulien Tolsma (Regional Public Health Service Brabant Zuid-Oost, Eindhoven, the Netherlands), Jacobien Veenemans (Admiraal De Ruyter Hospital, Goes, the Netherlands), Dewi van der Vegt (PAMM Laboratory for Pathology and Medical Microbiology, Veldhoven, the Netherlands), Martine Verelst (University Hospitals Leuven, Leuven, Belgium), Carlo Verhulst (Amphia Hospital, Breda, the Netherlands), Pascal De Waegemaeker (Ghent University Hospital, Ghent, Belgium), Veronica Weterings (Amphia Hospital, Breda, the Netherlands), Clementine Wijkmans (Regional Public Health Service Hart voor Brabant, Tilburg, the Netherlands), Patricia Willemse-Smits (Elkerliek Hospital, Helmond, the Netherlands), Ina Willemsen (Amphia Hospital, Breda, the Netherlands).

\section{ORCID IDs}

Herman Goossens https://orcid.org/0000-0003-4891-4031

Jan Kluytmans https://orcid.org/0000-0003-2665-6844

Marjolein Kluytmans-van den Bergh https://orcid.org/0000-0002-6576-6408

Christine Lammens https://orcid.org/0000-0001-9424-3107

Isabel Leroux-Roels https://orcid.org/0000-0002-5084-4696

Veroniek Saegeman http://orcid.org/0000-0003-0439-0205

Paul Savelkoul https://orcid.org/0000-0002-1318-2734

Ina Willemsen https://orcid.org/0000-0003-3112-6848

\section{REFERENCES}

1. European Centre for Disease Prevention and Control. Plasmid-mediated colistin resistance in Enterobacteriaceae. 2016. https://ecdc.europa.eu/sites/portal/files/media/en/publications/Publications/enterobacteriaceae- 
risk-assessment-diseases-caused-by-antimicrobial-resistant-microorganisms-europe-june2016.pdf (accessed December 10, 2019).

2. European Centre for Disease Prevention and Control. ECDC study protocol for genomic-based surveillance of carbapenem-resistant and/or colistin-resistant Enterobacteriaceae at the EU level. Version 2.0. 2018. https://ecdc.europa.eu/sites/portal/files/documents/Protocol-genomicsurveillance-resistant-Enterobacteriaceae-v2 0.pdf (accessed December 10, 2019).

3. European Medicines Agency. Updated advice on the use of colistin products in animals within the European Union: development of resistance and possible impact on human and animal health. EMA/CVMP/CHMP/231573/2016. 2016. https://www.ema.europa.eu/documents/scientificguideline/updated-advice-use-colistin-products-animals-within-european-union-developmentresistance-possible en-0.pdf (accessed December 10, 2019).

4. World Health Organisation. Critically important antimicrobials for human medicine, 5th revision. Geneva: World Health Organization, 2017. http://apps.who.int/iris/bitstream/handle/10665/255027/9789241512220eng.pdf;jsessionid=C254F1D8EB7988C52B0188B08CCAC3DC?sequence $=1$ (accessed December 10, 2019).

5. Nordmann P, Jayol A, Poirel L. A universal culture medium for screening polymyxin-resistant Gram-negative isolates. J Clin Microbiol 2016;54:1395-1399.

6. Matuschek E, Ahman J, Webster C, Kahlmeter G. Antimicrobial susceptibility testing for colistin evaluation of seven commercial MIC products against standard broth microdilution for Escherichia coli, Klebsiella pneumoniae, Pseudomonas aeruginosa, and Acinetobacter spp. Clin Microbiol Infect 2018;24:865-870.

7. European Committee on Antimicrobial Susceptibility Testing. Breakpoint tables for interpretation of MICs and zone diameters. Version 8.1. 2018.

http://www.eucast.org/fileadmin/src/media/PDFs/EUCAST files/Breakpoint tables/v 8.1 Breakpoi nt Tables.pdf (accessed December 10, 2019).

8. European Committee on Antimicrobial Susceptibility Testing. EUCAST guidelines for detection of resistance mechanisms and specific resistances of clinical and/or epidemiological importance. Version 2.0. 2017. http://www.eucast.org/fileadmin/src/media/PDFs/EUCAST files/Resistance mechanisms/EUCAS T detection of resistance mechanisms 170711.pdf (accessed December 10, 2019).

9. Wilkinson MD, Dumontier M, Aalbersberg IJ, et al. The FAIR guiding principles for scientific data management and stewardship. Sci Data 2016;3:160018.

10. Kluytmans-van den Bergh MFQ, Verhulst C, Willemsen LE, Verkade E, Bonten MJM, Kluytmans JAJW. Rectal carriage of extended-spectrum beta-lactamase-producing Enterobacteriaceae in hospitalized patients: selective pre-enrichment increases the yield of screening. J Clin Microbiol 2015;53:2709-2712.

11. Jazmati N, Hein R, Hamprecht A. Use of an enrichment broth improves detection of extendedspectrum-beta-lactamase-producing Enterobacteriaceae in clinical stool samples. J Clin Microbiol 2016;54:467-470. 
Figure 1. The Dutch-Belgian border region.

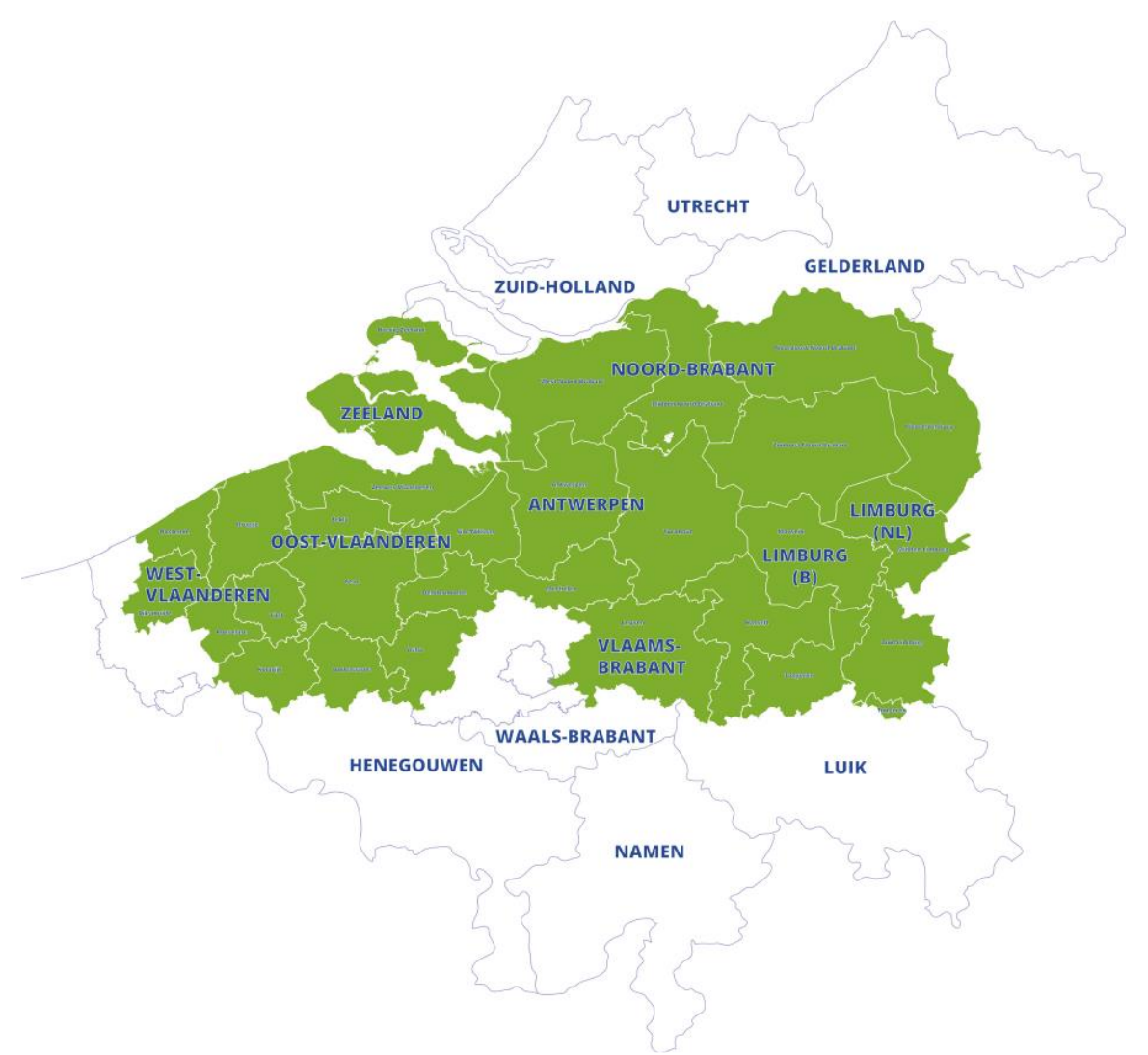

This figure was kindly provided by Interreg Flanders-The Netherlands. 
Figure 2. Timeline and anticipated numbers for microbiological sampling in the participating hospitals, nursing homes, daycare centres, primary schools, broiler farms and pig farms.

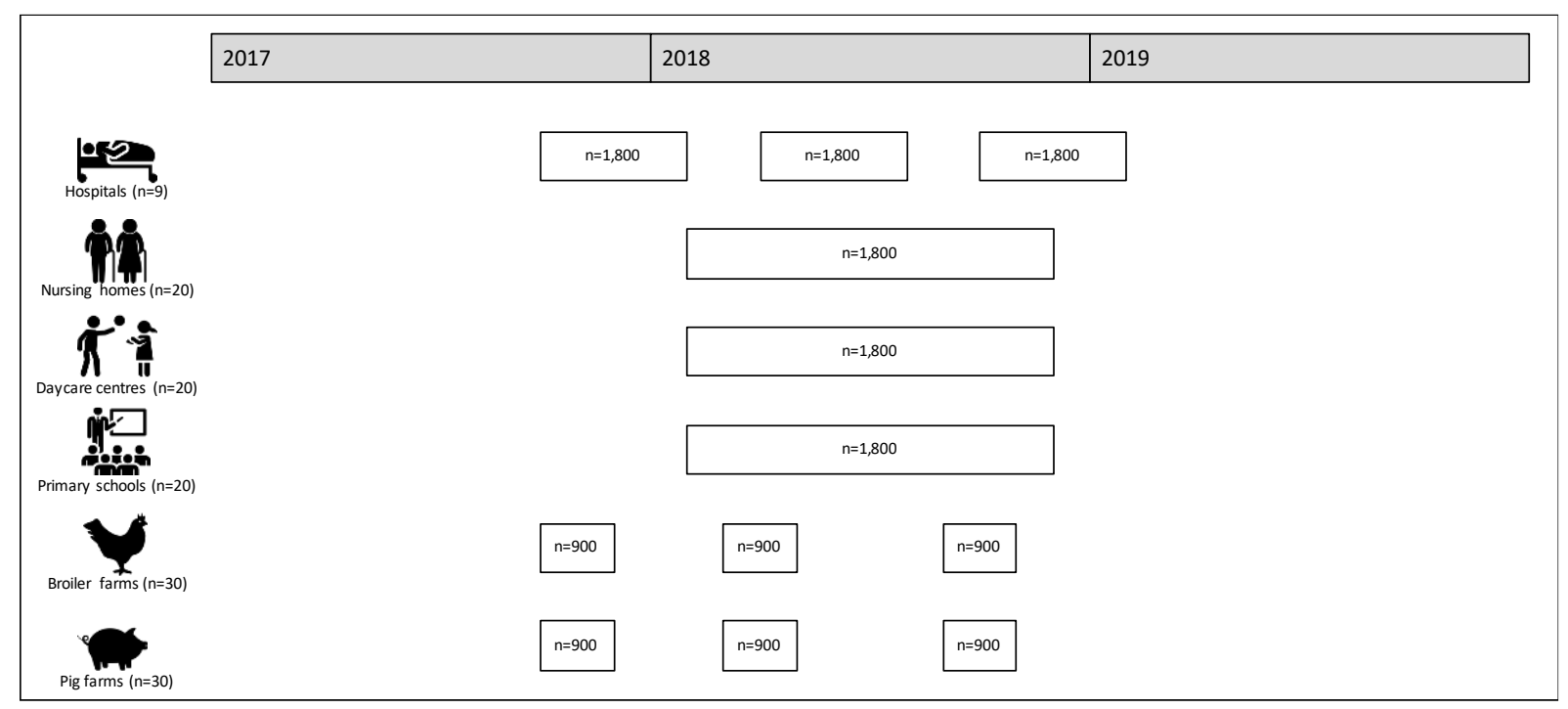


Figure 3. Flowchart of laboratory procedures.

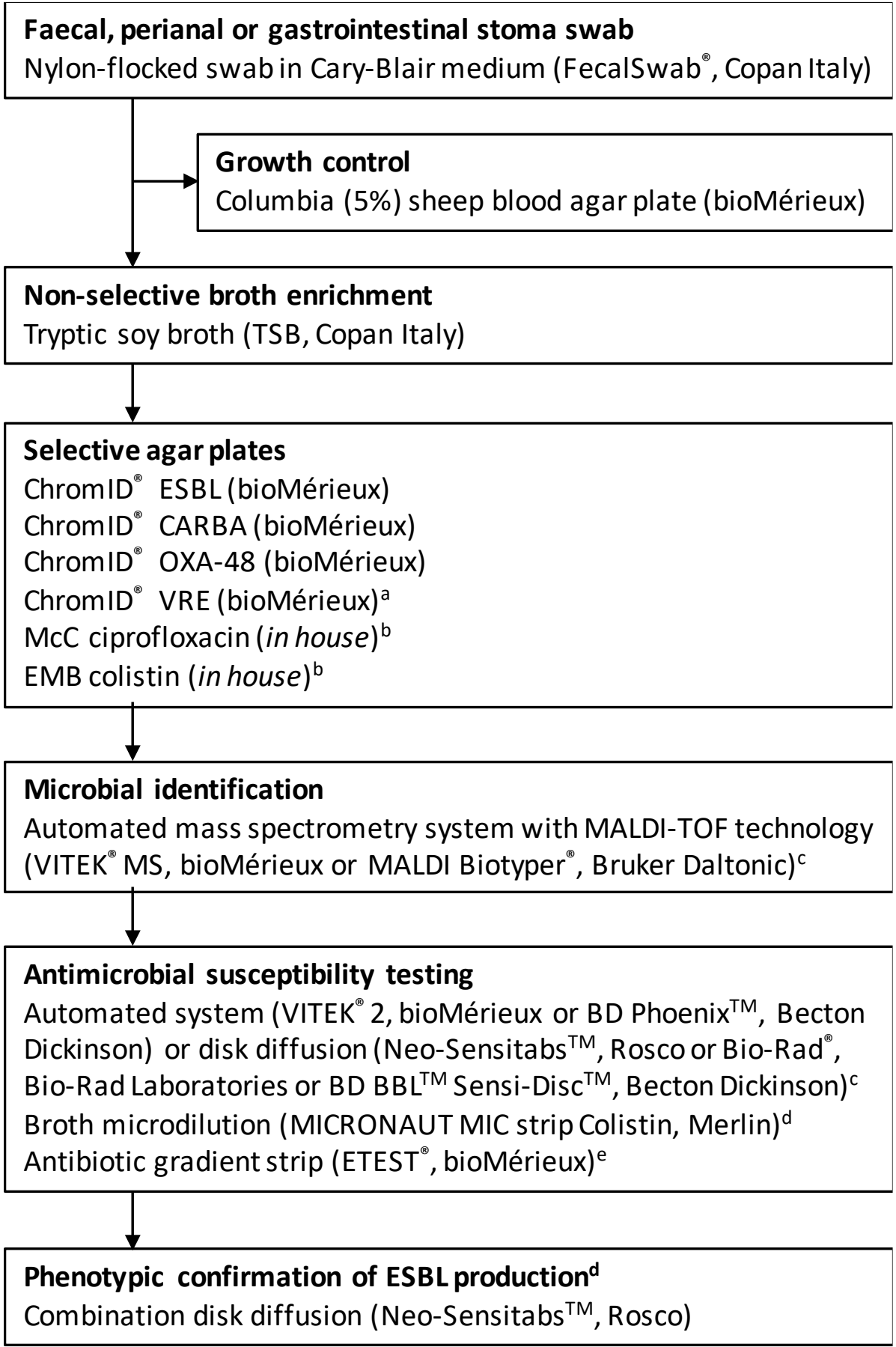

\footnotetext{
a. ChromID ${ }^{\circledR}$ VRE are used for human samples only.

b. McC ciprofloxacin and EMB colistin are used in three of nine laboratories (Table 1).

c. The method used is dependent on local routine laboratory practice (Table 1).

d. Broth microdilution is used to determine the MIC for colistin in Enterobacterales.

e. The antibiotic gradient strip method is used to determine the MICs for vancomycin and teicoplanin in enterococci.
} 
Figure 4. Structure of study numbers and isolate numbers.

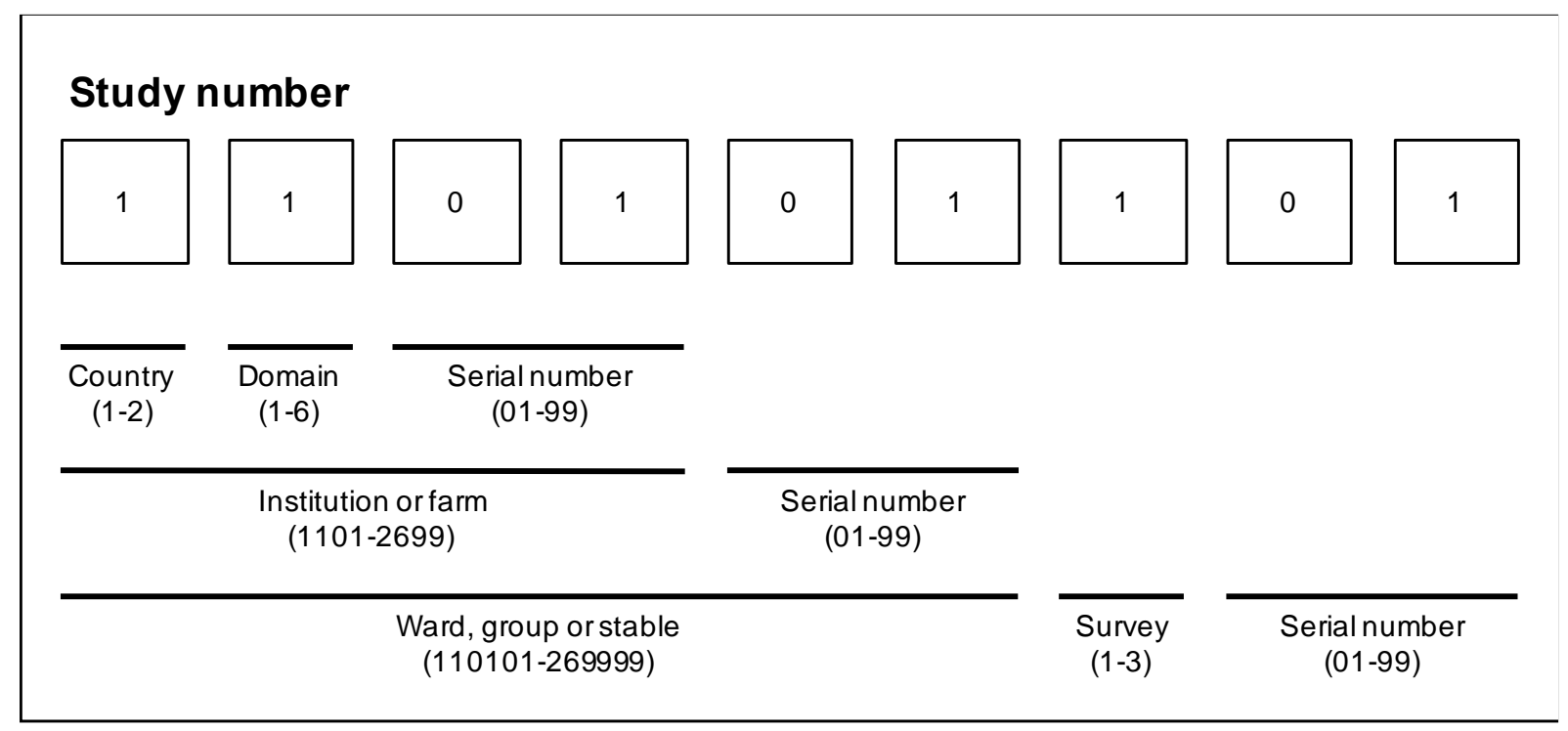

\section{Isolate number}
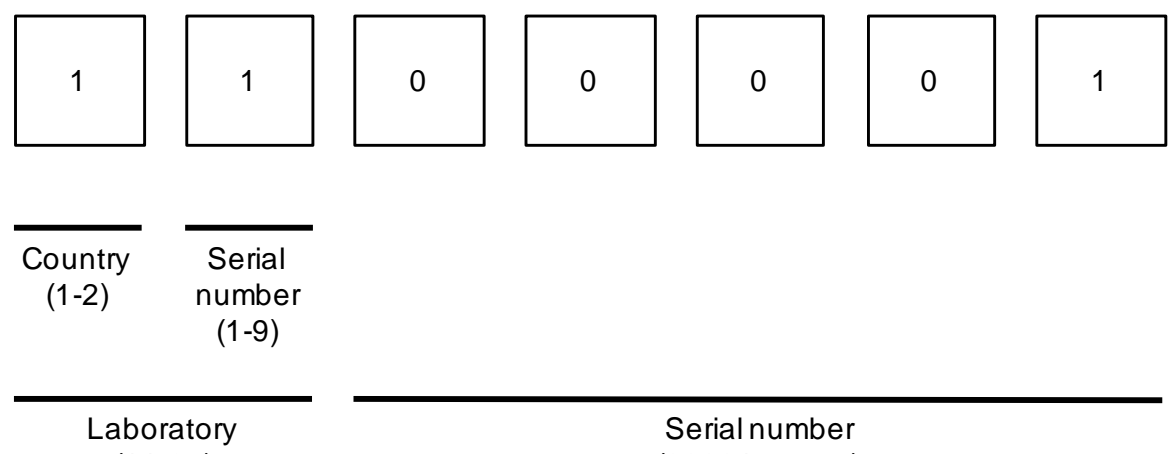

(11-29) 
Table 1. Laboratory methods used in the nine participating laboratories.

\begin{tabular}{|c|c|c|c|c|c|c|c|c|c|}
\hline & \multicolumn{9}{|c|}{ Laboratory } \\
\hline & $\mathrm{ADRH}$ & $\mathrm{AH}$ & AUH & ETH & GUH & MUMC & PAMM & UA & UHL \\
\hline \multicolumn{10}{|l|}{ Faecal, perianal or gastrointestinal stoma swab } \\
\hline FecalSwab ${ }^{\circledR}$ (Copan Italy) & $x$ & $x$ & $x$ & $x$ & $x$ & $x$ & $x$ & $x$ & $\mathrm{x}$ \\
\hline \multicolumn{10}{|l|}{ Growth control } \\
\hline Columbia (5\%) sheep blood agar plate (bioMérieux) & $\mathrm{x}$ & $\mathrm{x}$ & $\mathrm{x}$ & $\mathrm{x}$ & $\mathrm{x}$ & $\mathrm{x}$ & $\mathrm{x}$ & $\mathrm{x}$ & $\mathrm{x}$ \\
\hline \multicolumn{10}{|l|}{ Non-selective broth enrichment } \\
\hline Tryptic soy broth (Copan Italy) & $x$ & $x$ & $x$ & $\mathrm{x}$ & $x$ & $x$ & $x$ & $x$ & $x$ \\
\hline \multicolumn{10}{|l|}{ Selective agar plates } \\
\hline ChromID ${ }^{\circledR}$ ESBL (bioMérieux) & $\mathrm{x}$ & $\mathrm{x}$ & $\mathrm{x}$ & $\mathrm{x}$ & $\mathrm{x}$ & $\mathrm{x}$ & $\mathrm{x}$ & $\mathrm{x}$ & $\mathrm{x}$ \\
\hline ChromID ${ }^{\circledR}$ CARBA (bioMérieux) & $\mathrm{x}$ & $\mathrm{x}$ & $\mathrm{x}$ & $\mathrm{x}$ & $\mathrm{x}$ & $\mathrm{x}$ & $\mathrm{x}$ & $\mathrm{x}$ & $\mathrm{x}$ \\
\hline ChromID ${ }^{\circledR}$ OXA-48 (bioMérieux) & $x$ & $x$ & $x$ & $x$ & $x$ & $x$ & $x$ & $x$ & $x$ \\
\hline ChromID ${ }^{\circledR}$ VRE (bioMérieux) ${ }^{\mathrm{a}}$ & $\mathrm{x}$ & $\mathrm{x}$ & $\mathrm{x}$ & $\mathrm{x}$ & $\mathrm{x}$ & $\mathrm{x}$ & $\mathrm{x}$ & & $\mathrm{x}$ \\
\hline McC ciprofloxacin (in house) & & $\mathrm{x}$ & $\mathrm{x}$ & & & & & $\mathrm{x}$ & \\
\hline EMB colistin (in house) & & $\mathrm{x}$ & $\mathrm{x}$ & & & & & $\mathrm{x}$ & \\
\hline \multicolumn{10}{|l|}{ Microbial identification } \\
\hline VITEK ${ }^{\circledR}$ MS (bioMérieux) & $\mathrm{x}$ & $\mathrm{x}$ & & & & $\mathrm{x}$ & $\mathrm{x}$ & & \\
\hline MALDI Biotyper ${ }^{\circledR}$ (Bruker Daltonic) & & & $\mathrm{x}$ & $\mathrm{x}$ & $\mathrm{x}$ & & & $\mathrm{x}$ & $\mathrm{x}$ \\
\hline \multicolumn{10}{|l|}{ Antimicrobial susceptibility testing } \\
\hline VITEK ${ }^{\circledR} 2$ (bioMérieux) & $x$ & $x$ & & & & $x$ & $x$ & & $x^{c}$ \\
\hline BD Phoenix ${ }^{\mathrm{TM}}$ (bioMérieux) & & & & $x$ & & & & & \\
\hline Neo-Sensitabs ${ }^{\mathrm{TM}}$ (Rosco) & & & $x^{b}$ & & & & & $x^{b}$ & $x^{d}$ \\
\hline Bio-Rad $^{\circledR}$ (Bio-Rad Laboratories) & & & & & $x^{b}$ & & & & \\
\hline BD BBL ${ }^{\mathrm{TM}}$ Sensi-Disc ${ }^{\mathrm{TM}}$ (Becton Dickinson) & & & $\mathrm{x}$ & & & & & $\mathrm{x}$ & \\
\hline MICRONAUT MIC strip Colistin (Merlin)e & & $\mathrm{x}$ & $\mathrm{x}$ & & & & & $\mathrm{x}$ & \\
\hline 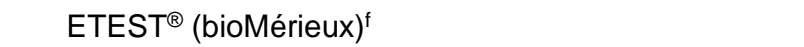 & $\mathrm{x}$ & $\mathrm{x}$ & $\mathrm{x}$ & $\mathrm{x}$ & $\mathrm{x}$ & $\mathrm{x}$ & $\mathrm{x}$ & & $\mathrm{x}$ \\
\hline \multicolumn{10}{|l|}{ Phenotypic confirmation of ESBL production } \\
\hline Neo-Sensitabs ${ }^{\top M}$ (Rosco) & $\mathrm{x}$ & $\mathrm{x}$ & $\mathrm{x}$ & $\mathrm{x}$ & $\mathrm{x}$ & $\mathrm{x}$ & $\mathrm{x}$ & $\mathrm{x}$ & $\mathrm{x}$ \\
\hline
\end{tabular}

ADRH=Admiraal De Ruyter Hospital; AH=Amphia Hospital; AUH=Antwerp University Hospital; ETH=Elisabeth-TweeSteden Hospital; GUH=Ghent University Hospital; MUMC=Maastricht University Medical Center+; PAMM=PAMM Laboratory for Pathology and Medical Microbiology; UA=University of Antwerp; UHL=University Hospitals Leuven.

a. ChromID ${ }^{\circledR}$ VRE is used for human samples only.

b. For all isolates that are selected for whole genome sequencing, antimicrobial susceptibility testing with VITEK ${ }^{\circledR} 2$ (bioMérieux) is additionally performed in Amphia Hospital.

c. For Enterobacterales, antimicrobial susceptibility testing is performed with VITEK ${ }^{\circledR} 2$ (bioMérieux).

d. For enterococci, antimicrobial susceptibility testing is performed with Neo-Sensitabs ${ }^{\mathrm{TM}}$ (Rosco).

e. MICRONAUT MIC strip Colistin (Merlin) is used to determine the MIC for colistin in Enterobacterales.

f. ETEST $^{\circledast}$ (bioMérieux) is used to determine the MICs for vancomycin and teicoplanin in enterococci. 
Table 2. Antimicrobial susceptibility testing in Enterobacterales.

\begin{tabular}{|c|c|c|c|}
\hline & \multicolumn{2}{|c|}{ Automated systems } & \multirow{2}{*}{$\begin{array}{c}\text { Disc diffusion method } \\
\text { Neo-Sensitabs } \\
\text { Disc }^{\mathrm{TM}} \text { / Bio-Rantent (ug) }\end{array}$} \\
\hline & $\begin{array}{l}V_{i t e k^{\circledR}} 2-\mathrm{N} 344 \\
\text { MIC range (mg/L) }\end{array}$ & $\begin{array}{l}\text { BD Phoenix }{ }^{\mathrm{TM}}-\mathrm{NMIC}-417 \\
\text { MIC range (mg/L) }\end{array}$ & \\
\hline Amikacin & & $4-16$ & 30 \\
\hline Amoxicillin/clavulanic acid & 2/2-32/2 & 2/2-32/2 & $20 / 10$ \\
\hline Ampicillin & $2-32$ & $2-8$ & 10 \\
\hline Aztreonam & & & 30 \\
\hline Cefepime & & $1-16$ & 30 \\
\hline Cefotaxime & $0.25-64$ & & 5 \\
\hline Cefoxitin & $4-64$ & $4-16$ & 30 \\
\hline Ceftazidime & $0.12-64$ & $0.5-16$ & 10 \\
\hline Ceftriaxone & & $0.5-4$ & 30 \\
\hline Cefuroxime & $1-64$ & $2-8$ & 30 \\
\hline Ciprofloxacin & $0.25-4$ & $0.25-1$ & 5 \\
\hline Colistin & $0.5-16$ & $1-4$ & \\
\hline Ertapenem & & $0.25-1$ & \\
\hline Fosfomycin & $16-256$ & $16-128$ & 200 \\
\hline Gentamicin & $1-16$ & $1-4$ & \\
\hline Imipenem & $0.25-16$ & $0.25-8$ & \\
\hline Levofloxacin & & $0.5-2$ & 5 \\
\hline Meropenem & $0.25-16$ & $0.125-8$ & 10 \\
\hline Nitrofurantoin & $16-512$ & $16-64$ & 100 \\
\hline Norfloxacin & & $0.5-2$ & \\
\hline Piperacillin/tazobactam & $4 / 4-128 / 4$ & $4 / 4-64 / 4$ & $30 / 6$ \\
\hline Temocillin & & & 30 \\
\hline Tigecyclin & & $0.5-2$ & \\
\hline Tobramycin & $1-16$ & $1-4$ & 10 \\
\hline Trimethoprim & $0.5-16$ & $1-4$ & \\
\hline Trimethoprim/sulfamethoxazole & $1 / 19-16 / 304$ & $1 / 19-4 / 76$ & $1.25 / 23.75$ \\
\hline
\end{tabular}


Table 3. Antimicrobial susceptibility testing in enterococci.

\begin{tabular}{|c|c|c|c|}
\hline & \multicolumn{2}{|c|}{ Automated systems } & \multirow{2}{*}{ 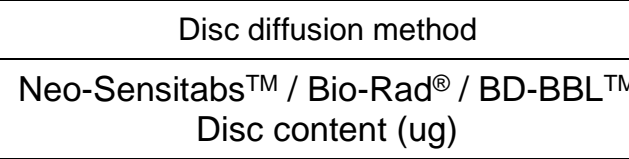 } \\
\hline & $\begin{array}{c}\text { Vitek }{ }^{\circledR 2} \text { - P586 } \\
\text { MIC range (mg/L) }\end{array}$ & $\begin{array}{l}\text { BD Phoenix }{ }^{\mathrm{TM}} \text { - PMIC-96 } \\
\text { MIC range }(\mathrm{mg} / \mathrm{L})\end{array}$ & \\
\hline Ampicillin & $2-32$ & $1-16$ & 2 \\
\hline Ampicillin/sulbactam & $2 / 1-32 / 16$ & & \\
\hline Clindamycin & $0.25-8$ & & \\
\hline Erythromycin & $0.25-8$ & & \\
\hline Fosfomycin & & $16-64$ & 200 \\
\hline Gentamycin high-level (synergy) & 500 & 500 & \\
\hline Imipenem & $1-16$ & $1-8$ & \\
\hline Levofloxacin & & $0.5-4$ & \\
\hline Linezolid & $0.5-8$ & $0.5-4$ & 10 \\
\hline Moxifloxacin & $0.25-8$ & & \\
\hline Nitrofurantoin & $16-512$ & $16-64$ & \\
\hline Quinupristin / dalfopristin & $0.25-16$ & & \\
\hline Streptomycin high-level (synergy) & 1000 & & \\
\hline Teicoplanin & $0.5-32$ & $0.5-8$ & \\
\hline Tetracyclin & $1-16$ & $0.5-2$ & \\
\hline Tigecyclin & $0.12-2$ & $0.125-1$ & 15 \\
\hline Trimethoprim & & $0.5-4$ & \\
\hline Trimethoprim/sulfamethoxazole & $0.5 / 9.5-16 / 304$ & $0.5 / 9.5-4 / 76$ & \\
\hline Vancomycin & $0.5-32$ & $0.5-8$ & 5 \\
\hline
\end{tabular} \\ 216.v1}


Table 4. Interpretation rules for the phenotypic confirmation of extended-spectrum beta-lactamase production in Enterobacterales. ${ }^{8}$

\begin{tabular}{|c|c|c|c|c|c|}
\hline \multirow[b]{2}{*}{ Enterobacterales } & \multirow[b]{2}{*}{$\begin{array}{l}\text { MIC cefoxitin } \\
(\mathrm{mg} / \mathrm{L})\end{array}$} & \multicolumn{3}{|c|}{ Combination disk diffusion - difference in inhibition zone } & \multirow[b]{2}{*}{ Conclusion } \\
\hline & & $\begin{array}{c}\text { CTX-Cc }- \text { CTX } 30^{d} \\
(\mathrm{~mm})\end{array}$ & $\begin{array}{c}\text { CAZ-Ce - CAZ30 } \\
(\mathrm{mm})\end{array}$ & $\begin{array}{c}\text { FEP-Cg - FEP30 } \\
(\mathrm{mm})\end{array}$ & \\
\hline \multirow{6}{*}{ Group $1^{a}$} & - & $>=5$ & $>=5$ & - & ESBL positive \\
\hline & - & $>=5$ & $<5$ & - & ESBL positive \\
\hline & - & $<5$ & $>=5$ & - & ESBL positive \\
\hline & $>=16$ & $<5$ & $<5$ & $>=5$ & ESBL positive \\
\hline & $<16$ & $<5$ & $<5$ & $>=5$ & ESBL negative \\
\hline & - & $<5$ & $<5$ & $<5$ & ESBL negative \\
\hline \multirow{2}{*}{ Group $2^{b}$} & - & - & - & $>=5$ & ESBL positive \\
\hline & - & - & - & $<5$ & ESBL negative \\
\hline
\end{tabular}

a. Group 1 Enterobacterales (no inducible chromosomal AmpC) include Citrobacter amalonaticus, Citrobacter farmeri, Citrobacter gillenii, Citrobacter koseri (diversus), Citrobacter sedlakii, Escherichia coli, Escherichia fergusonii, Escherichia hermannii, Klebsiella pneumoniae, Klebsiella oxytoca, Kluyvera ascorbata, Leclercia adecarboxylata, Proteus mirabilis, Proteus penneri, Proteus vulgaris, Rahnella aquatilis, Raoultella spp., Salmonella spp., and Shigella spp.

b. Group 2 Enterobacterales (inducible chromosomal AmpC) include Citrobacter braakii, Citrobacter freundii, Citrobacter werkmanii, Citrobacter youngae, Citrobacter murliniae, Enterobacter cloacae (complex), Hafnia alvei, Klebsiella aerogenes, Morganella morganii, Pantoea agglomerans, Providencia spp., Serratia spp., and Yersinia spp.

c. Neo-Sensitabs ${ }^{\text {TM }}$ CTX-C contains cefotaxime 30 ug and clavulanic acid 10 ug.

d. Neo-Sensitabs ${ }^{\mathrm{TM}}$ CTX30 contains cefotaxime 30 ug.

e. Neo-Sensitabs ${ }^{\mathrm{TM}}$ CAZ-C contains ceftazidime $30 \mathrm{ug}$ and clavulanic acid $10 \mathrm{ug}$.

f. Neo-Sensitabs ${ }^{\mathrm{TM}}$ CAZ30 contains ceftazidime $30 \mathrm{ug}$.

g. Neo-Sensitabs ${ }^{\mathrm{TM}}$ FEP-C contains cefepime $30 \mathrm{ug}$ and clavulanic acid $10 \mathrm{ug}$.

h. Neo-Sensitabs ${ }^{\text {TM }}$ FEP30 contains cefepime 30 ug. 